\title{
Psychological factors of social anxiety in Russian adolescents
}

\author{
Tatiana S. Pavlova ${ }^{a \star}$, Alla B. Kholmogorova ${ }^{\text {b, }}$ \\ ${ }^{a}$ Center for Emergency Psychological Aid, Moscow State University of Psychology and Education, \\ Moscow, Russia \\ ${ }^{\mathrm{b}}$ Faculty of Psychological Counseling, Moscow State University of Psychology and Education, \\ Moscow, Russia \\ ${ }^{c}$ Research Institute of Psychiatry, Federal Medical Research Center for Psychiatry and Narcology \\ of the Ministry of Health of the Russian Federation, Moscow, Russia \\ *Corresponding author. E-mail: darzo@yandex.ru
}

Background. Social anxiety is one of the most common and disturbing conditions of childhood and adolescence. It is defined as an excessive fear of embarrassment or humiliation in social performance situations. Recent studies have identified a number of psychological factors that could explain the maintenance of the condition.

Objective. The objective of this study was to investigate psychological factors of social anxiety in adolescents with a multifactor psychosocial model.

Design: The study population comprised 183 Russian-speaking adolescents from Moscow secondary schools, ranging in age from 12 to 16 years. Self-report measures were used to access social anxiety, symptoms of depression, gender role identification, perfectionism, hostility, family emotional communications, and social support.

Results. The results indicate that social anxiety was positively correlated with symptoms of depression and suicidal thoughts. No quantitative differences in social anxiety between girls and boys were found, while masculinity and undifferentiated gender identification had a strong association with social anxiety. A positive correlation was found between "concern over mistakes" (fear of making a mistake and being negatively compared with peers) and "overdoing" (spending too much time doing homework and too little or none communicating with peers), using the Child Perfectionism Questionnaire (CPQ) subscales and Social Anxiety and Distress Scale (SADS) total score. Positive correlations were found between social anxiety and suppression of emotions and outward well-being subscales, as well in as the Family Emotional Communication (FEC) total score. It is not common to discuss emotions and feelings; it is difficult to share negative experiences; and it is important for the families of socially anxious adolescents to put up a good front. Analysis revealed significant negative correlations between the SADS total score (as well its subscales) and the Social Support scale total score and emotional support subscale; the Practical Support scale negatively correlated with the SADS total score. 
Conclusion. These findings confirm our hypothesis that social anxiety has a complex nature and can be dealt with by a multifactor psychosocial model.

Keywords: social anxiety, adolescents, gender, perfectionism, family

\section{Introduction}

Social anxiety is a widespread and rather disturbing condition. It is defined as an excessive fear of embarrassment or humiliation in social performance situations. Its negative impact results in long-term functional impairment, especially in adolescence (Bruch, Fallon, \& Heimberg, 2003; Dryman, Gardner, Weeks, \& Heimberg, 2016; Peleg, 2012). While it is well known that social anxiety is a highly prevalent condition in Western countries, its epidemiology in Russia remains unclear, partly due to a lack of assessment methods and partly to a lack of research. Epidemiological Catchment Area study data shows a bimodal distribution of age of onset, with the first peak occurring before 5 years of age and a second peak occurring around 13 years (Schneier, Johnson, Hornig, Liebowitz, \& Weissman, 1992). Social anxiety in adolescence can result in mood disorders in adulthood. Therefore it is especially important to learn more about factors contributing to this condition in teenagers.

Many factors are known to trigger social anxiety and play a role in its development, and there are several models describing the interaction of these factors. Cognitive models emphasize dysfunctional beliefs and attention bias (Clark \& Wells, 1995; Heimberg, Brozovich, \& Rapee, 2014). Most typical cognitions for the socially anxious are that others are overly critical and that social situations are inherently dangerous; therefore, perfect social performance is needed in order to avoid rejection. Clark and Wells suggest that self-focused attention is the central issue that generates anxiety during an interaction and that post-event processing is the key process that maintains it over time. Mindfulness is gaining popularity as an important strategy in overcoming social anxiety and shows promising results in reducing post-event processing (Shikatani, Antony, Kuo, \& Cassin, 2014; Bockstaele \& Bögels, 2014). The interpersonal approach emphasizes a self-perpetuating interpersonal cycle, social developmental experiences, a social skills deficit, and a self-protective strategy that is based in early childhood during communication with significant others (Alden \& Taylor, 2004). An evolutionary perspective on social anxiety relies on Nesse's works on adaptive functions of negative emotions in general, and fear and anxiety in particular. While anxiety may be painful and disruptive for those experiencing it, it also helps to anticipate and avoid danger. People are particularly sensitive to social stimuli, as they belong to a social species, and for a long time, survival of the individual depended on affiliation with the group and social status in this group, so some may be overly sensitive to any threat to social status, exclusion from or rejection by the group (Gilbert \& Trower, 2001). A parent-child interaction model of social anxiety disorders in youth specifies five risk factors: temperamental characteristics of the child, parental anxiety, attachment processes in the parent-child dyad, information-processing biases, and parenting practices (Ollendick \& Benoit, 2012). According to a diathesis-stress model of childhood shyness, the combination of biological diathesis (genetic variation in neurochemical and physiological systems) and psychological stress (family, school, 
and peer environment) results in shyness in children, which later sometimes leads to social withdrawal, social phobia, and depression in adolescence and adulthood (Schmidt, Polak, \& Spooner, 2005).

S.G. Hofmann (2007) proposed another way of explaining the symptoms and etiology of social anxiety. This model assumes that social fears and anxiety are associated with unrealistically high social standards and poorly defined social goals. When individuals with social anxiety confront social situations, they shift their attention towards their anxiety, create a negative image of themselves as social objects, and overestimate the likelihood and consequences of social mishaps. In order to avoid these expected mishaps, they revert to such maladaptive coping strategies as avoidance and safety behaviors. Social interaction is followed by post-event rumination, which leads to further social apprehension in the future.

A multifactor psychosocial model of affective disorders as a methodological basis for social anxiety research in adolescence. We relied on a recently developed multifactor psychosocial model of affective disorders (Kholmogorova, 2011). A multifactor psychosocial model of affective disorders can serve as a theoretical basis for social anxiety research, as it provides an opportunity to integrate different doctrines in one perspective. So far, a series of studies has been conducted in this framework, whereby factors of mood disorders development are grouped into four levels: macrosocial, family, interpersonal, and personal.

The macrosocial level describes social and economic instability and its consequences, such as a growing number of disadvantaged families and unprecedented growth of social orphanhood, which results in an increasing number of traumatized children who are especially vulnerable to emotional disturbances. Society's high standards of personal success and perfection, exaggerated educational requirements for children that are hardly consistent with their physical and emotional development, elevated standards of physical appearance promoted by the mass media, all play a role in lowering children's self-esteem. Gender stereotypes are still powerful in modern Russian society. The cult of masculinity and male emotional inhibition results in substance abuse, risk-taking behavior, and reluctance to seek professional help among the male population.

The family level is described by the family system's characteristics: structure, macro- and micro dynamics, and ideology. Characteristics such as diffuse personal boundaries, criticism, parental control, perfectionistic standards, perception of the world as hostile and/or disapproving, insecure attachment types, history of severe diseases, sudden death, physical or sexual abuse in the family, may serve as a basis for development of a negative cognitive scheme on the personal level. This scheme is characterized by such dysfunctional beliefs and attitudes as: "the world is dangerous", "I am weak", or "other people are hostile". Therefore a person's own hostility and mistrust are attributed to others, in turn provoking negative expectations from the others.

On the interpersonal level, these beliefs result in a narrowing social network and lack of social support. Consequently anxiety increases even more, serving as an additional factor in developing an anxiety disorder, and therefore creating a vicious circle of anxiety. Social support is a non-specific protective factor for psychological well-being: A deficit in close interpersonal connections and a formal approach to 
communication lead to emotional ill-being and social maladjustment. Socially anxious people have been shown to have fewer dating and sexual relationships, fewer friends, and to be less likely to marry than people in the general population or even patients with other anxiety disorders (Hart, Turk, Heimberg, \& Liebowitz, 1999).

The present study aimed at addressing these research questions and examined complex psychological factors associated with social anxiety.

We believe that this model of social anxiety can contribute to developing a holistic picture of the condition. Therefore, the aim of this research was a complex investigation of psychological factors of social anxiety in adolescence.

\section{Method}

\section{Participants}

Participants were 183 teenagers (49\% boys) from seventh to tenth grade from secondary schools in Moscow. They ranged in age from 12 to 16. The children participated voluntarily during their school hours. Filling out the questionnaires took around 50 minutes. A lecture on social anxiety in teenagers was given to participants' parents after the screening.

\section{Measures}

The Social Avoidance and Distress Scale (SADS, 1969) is a 28-item self-report inventory with a true/false format. It consists of two separate factors, Social Distress and Social Avoidance. In a sample of university students, internal consistency was high - the Kuder-Richardson formula was 0.94 (Watson \& Friend, 1969). Recent studies have demonstrated the reliability and validity of the SADS for the Russianspeaking youth population (Krasnova, 2013).

The Children's Depression Inventory (CDI; M. Kovacs, 1992) is a 27-item self-report measure assessing children's depressive symptoms. Each item consists of three sentences describing a symptom of depression, in increasing severity. Respondents choose the sentence that best describes them during the past week. Each item set is scored from 0 (symptom is absent) to 2 (symptom is always or nearly always present). Scores are summed across all items. Higher scores indicate more depressive symptoms. The questionnaire consists of five scales: "Anhedonia", "Negative SelfEsteem", "Ineffectiveness", "Interpersonal Problems", and "Negative Mood".

Previous studies have demonstrated that the CDI has good internal consistency (a coefficient of 5.86). This study used the Total Depression score as well as subscales scores. Suicidal ideation: Similar to past research (Vinas, 2002), suicidal ideation was assessed using item 9 from the CDI, which requires the respondent to endorse one of the following three choices: (0) I do not think about killing myself"; (1) I think about killing myself, but I would not do it; or (2) I want to kill myself. Scores of 1 or 2 on this item, based on the second or third response, respectively, were used to indicate suicidal ideation.

The Masculinity and Femininity scale (Dvoryanchikov, 2011), modified from S. Bem Sex-Role Inventory (for Russian adaptation see Bessonova, 1994), characterizes individuals as masculine, feminine, androgynous, or undifferentiated. Respondents rate on a 4 -point Likert scale the extent to which characteristics (tradi- 
tionally masculine or feminine mixed with neutral ones) are descriptive of them. For example, masculine characteristics include "brave", "determined", "intrusive", while feminine ones may be "gentle", "neat", "weak"; in addition the list includes N neutral items such as "sensible".

The Child Perfectionism Questionnaire (CPQ, Volikova, 2012) measures different aspects of perfectionism: children's attitudes towards their studies, free time activities, peer relationships, and their appearance. This self-report questionnaire includes six scales: high personal standards (e.g., "I aim to be the best student in the class"), parental perfectionism (e.g., "I often hear from my parents that I have to meet their expectations"), concern over mistakes (e.g., "I feel very anxious while completing writing tasks at school, as I am afraid to make a mistake"), success as a main motive (e.g., "I have to be successful in order to feel good about myself"), physical perfectionism (e.g., "I often worry that my appearance is not perfect"), overdoing (e.g., "I do not have free time to hang around with my friends, as I am very busy with my lessons"). Internal consistency was good $(\alpha=.76)$.

The Hostility Test (Garanian, Kholmogorova, \& Udeeva, 2003) measures hostility, defined as a lasting inclination to attribute negative traits to social objects (e.g., dominating, tending to humiliate others, envious, cold, indifferent, malicious). Respondents are instructed to imagine that they are conducting an anonymous opinion poll; their task is to predict its results, giving the percent of those who agreed with the test statements. It contains three subscales: (1) perceiving others as "inclined to demonstrate their superiority and humiliate", for instance, "When I see other people's weaknesses and failures, it makes me feel better about myself"; (2) perceiving others as "despising weakness" (e.g., "One has to hide his weaknesses because other people can take advantage of them to show their superiority"); (3) perceiving others as cold and indifferent (e.g., "I feel it as a burden when someone asks me for help"). Internal consistency was good ( $\alpha=.79)$, Guttman split-half coefficient was 0.681 .

Family Emotional Communications (Kholmogorova \& Volikova, 2004) is a 33item questionnaire designed to access family emotional communications and intensity of negative emotions. Responses are coded on a 4-point Likert scale (ranging from $1=$ Absolutely agree to $4=$ Absolutely disagree). It contains eight subscales: parental criticism, concern with danger and failure, suppression of emotions, trying to put up a good front, fixation on negative feelings, provoked lack of trust in people, parental perfectionism, parental emotional dependence.

The Social Support Questionnaire F-SOZU-22 (E. Sommer, G. Fydrich, adapted to Russian sample by A. Kholmogorova, G. Petrova (2007)) is a 54-item questionnaire that assesses perceived social support using four subscales: (1) emotional support, (2) practical support, (3) social integration, and (4) social support satisfaction. Reliability coefficients and construct validity show satisfying results. Internal consistency was good $(\alpha=.79)$. Translated and adapted to a Russian sample by A. Kholmogorova, N. Garanian, and G. Petrova.

\section{Data analysis}

The statistical analysis was performed with the SPSS (version 11.0) program. The differences of mean scores were calculated using Mann-Whitney U nonparametric 
analysis. In assessment of correlations between the scales, the Spearman correlation coefficient was used. We also utilized a multiple regression model to test the hypothesized interaction effects.

\section{Results}

Prevalence of social anxiety symptoms and their connection with symptoms of depression and suicidal thoughts

In our sample no difference was found in social anxiety symptoms between girls and boys. Sixty-three percent reported moderate SA, 30\% reported medium SA, and high SA was found in $7 \%$ of participants.

\section{Depressive symptoms and social anxiety}

The Mann-Whitney U test showed statistically significant differences for depressive symptoms depending on the intensity of social anxiety in groups with low $(\mathrm{M}=48.7, \mathrm{SD}=6.5)$, medium $(\mathrm{M}=51.7, \mathrm{SD}=6.3)$, and high $(\mathrm{M}=66, \mathrm{SD}=13.9)$ SADS scores. It was the same with the CDI subscales and the SADS total score.

Table 1. Mean scores of the CDI subscales in adolescents with low, medium, and high social anxiety

\begin{tabular}{|c|c|c|c|}
\hline CDI subscales & $\begin{array}{c}\text { Group with low } \\
\text { social anxiety } \\
\text { M } \\
\text { (SD) }\end{array}$ & $\begin{array}{c}\text { Group with medium } \\
\text { social anxiety } \\
\text { M } \\
\text { (SD) }\end{array}$ & $\begin{array}{c}\text { Group with high } \\
\text { social anxiety } \\
\text { M } \\
\text { (SD) }\end{array}$ \\
\hline Anhedonia & $\begin{array}{c}49.7^{\mathrm{a}, \mathrm{b}} \\
(6.5)\end{array}$ & $\begin{array}{l}\mathbf{5 1 . 9}^{\mathbf{c}} \\
(7.4)\end{array}$ & $\begin{array}{c}\mathbf{6 0 . 7}^{\mathbf{b}, \mathrm{c}} \\
(9.3)\end{array}$ \\
\hline Negative self-esteem & $\begin{array}{l}\mathbf{5 2 . 5}^{\mathbf{b}} \\
(6.3)\end{array}$ & $\begin{array}{l}\mathbf{5 2 . 6}^{\mathbf{c}} \\
(9.3)\end{array}$ & $\begin{array}{l}\mathbf{6 5 . 7}^{\mathbf{b}, \mathbf{c}} \\
(17.1)\end{array}$ \\
\hline Ineffectiveness & $\begin{array}{l}49.7^{b} \\
(9.6)\end{array}$ & $\begin{array}{c}52 \\
(10.2)\end{array}$ & $\begin{array}{c}\mathbf{6 1 . 1}^{\mathbf{b}} \\
(14)\end{array}$ \\
\hline Interpersonal problems & $\begin{array}{c}\mathbf{4 6 . 9}^{\mathbf{b}} \\
(7)\end{array}$ & $\begin{array}{l}\mathbf{4 9 . 1}^{\mathrm{c}} \\
(5.3)\end{array}$ & $\begin{array}{l}\mathbf{5 8 . 9}^{\mathbf{b}, \mathbf{c}} \\
(11.2)\end{array}$ \\
\hline Negative mood & $\begin{array}{c}\mathbf{4 7 . 3}^{\mathbf{a}, \mathbf{b}} \\
(7.9)\end{array}$ & $\begin{array}{c}\mathbf{5 0 . 8}^{\mathbf{a}, \mathbf{c}} \\
(7.9)\end{array}$ & $\begin{array}{c}\mathbf{6 3 . 8}^{\mathbf{b}, \mathrm{c}} \\
(15.9)\end{array}$ \\
\hline
\end{tabular}

a - differences between groups with low and medium social anxiety are statistically significant (Mann-Whitney U criterion);

b - differences between groups with low and high social anxiety are statistically significant (MannWhitney U criterion);

c - differences between groups with medium and high social anxiety are statistically significant (Mann-Whitney U criterion).

\section{Social anxiety and suicidal intentions}

Findings suggest that the social distress and social avoidance scales of SADS, including the total score, were significantly related $(\mathrm{p} \leq 0.001)$ to the intensity of suicidal intentions (assessed by the CDI item "presence of suicidal thoughts"). 
Table 2. Results of multiple regression analyses with parameters of social anxiety and social support as independent variables and suicidal thoughts as the dependent variable

\begin{tabular}{lcccc}
\hline \multicolumn{1}{c}{$\begin{array}{c}\text { Independent } \\
\text { variables }\end{array}$} & $\begin{array}{c}\text { Dependent } \\
\text { variable }\end{array}$ & $\mathbf{B}$ & $\mathbf{T}$ & $\mathbf{R}^{\mathbf{2}}$ \\
\hline Social distress & & $\mathbf{. 5 6 8}$ & $\mathbf{4 . 3 8 6}$ & $\mathbf{. 0 0 0}$ \\
Social avoidance & & .117 & .876 & .385 \\
Emotional support & Suicidal & -.330 & -.788 & $\mathbf{. 0 8 0}$ \\
Practical support & thoughts & .058 & .322 & .749 \\
Social integration & & .088 & .561 & .577 \\
Social support satisfaction & & -.065 & -.633 & .530 \\
\hline
\end{tabular}

\section{Gender and social anxiety}

The Masculinity and Femininity scale enables us to characterize participants as masculine, feminine, androgynous, or undifferentiated. Gender role orientation distribution was analyzed in boys and girls. In both sexes, the androgynous type was prevalent ( $48 \%$ of boys and $36 \%$ of girls). As expected, the feminine type prevailed in girls (33\% of girls, $12 \%$ of boys) and the masculine type in boys (26\% of boys, $17 \%$ of girls). The undifferentiated type was found in $14 \%$ of both boys and girls.

Preliminary analysis using Mann-Whitney $U$ nonparametric analysis failed to find a significant difference between the males' $(\mathrm{M}=6.6, \mathrm{SD}=4.6)$ and females' $(\mathrm{M}=6.7, \mathrm{SD}=3.5)$ total scores and subscales on the SADS.

Table 3. Mean scores of the SADS subscales in adolescents with masculine, feminine, androgynous, or undifferentiated gender role orientations

\begin{tabular}{|c|c|c|c|c|}
\hline \multirow{3}{*}{$\begin{array}{l}\text { Social avoidance } \\
\text { and distress scale }\end{array}$} & \multicolumn{4}{|c|}{ Gender role orientation } \\
\hline & $\begin{array}{l}\text { androgynous } \\
\quad(N=55)\end{array}$ & $\begin{array}{c}\text { masculine } \\
(N=28)\end{array}$ & $\begin{array}{c}\text { feminine } \\
(N=35)\end{array}$ & $\begin{array}{l}\text { undifferentiated } \\
\qquad(N=21)\end{array}$ \\
\hline & $\begin{array}{c}\text { M } \\
(\mathrm{SD})\end{array}$ & $\begin{array}{c}\text { M } \\
(\mathrm{SD})\end{array}$ & $\begin{array}{c}\text { M } \\
(\mathrm{SD})\end{array}$ & $\begin{array}{c}\mathbf{M} \\
(\mathrm{SD})\end{array}$ \\
\hline Total score & $\begin{array}{l}5.9^{\mathrm{a}, \mathrm{c}} \\
(3.3)\end{array}$ & $\begin{array}{c}\mathbf{4 . 2}^{\mathrm{a}, \mathrm{b}, \mathrm{d}} \\
(2.5)\end{array}$ & $\begin{array}{l}\mathbf{8 . 1}^{\mathrm{b}, \mathrm{c}} \\
(5.1)\end{array}$ & $\begin{array}{c}8^{8.8^{\mathrm{d}}} \\
(6)\end{array}$ \\
\hline Social distress & $\begin{array}{c}3^{\mathrm{a}, \mathrm{c}} \\
(2.1)\end{array}$ & $\begin{array}{c}1.7^{\mathrm{a}, \mathrm{b}, \mathrm{d}} \\
(1.3)\end{array}$ & $\begin{array}{l}\mathbf{4 . 2}^{\mathrm{b}, \mathrm{c}} \\
(3.1)\end{array}$ & $\begin{array}{l}4.2^{\mathrm{d}} \\
(3.4)\end{array}$ \\
\hline Social avoidance & $\begin{array}{l}2.8^{\mathrm{c}} \\
(1.6)\end{array}$ & $\begin{array}{l}2.4^{\mathrm{b}} \\
(1.6)\end{array}$ & $\begin{array}{l}3.8^{\mathbf{b}, \mathbf{c}} \\
(2.4)\end{array}$ & $\begin{array}{c}4.5 \\
(3.1)\end{array}$ \\
\hline
\end{tabular}

a - differences between groups with androgynous and masculine gender role orientation are statistically significant (Mann-Whitney U criterion);

b - differences between groups with masculine and feminine gender role orientation are statistically significant (Mann-Whitney U criterion);

c - differences between groups with androgynous and feminine gender role orientation are statistically significant (Mann-Whitney U criterion);

d-differences between groups with masculine and undifferentiated gender role orientation are statistically significant (Mann-Whitney U criterion). 
Masculinity was found to be negatively correlated with social anxiety $(\mathrm{p} \leq 0.001)$. Lower social anxiety symptoms referred to a more masculine identity in boys and girls regardless of biological sex. Correlations between femininity and social anxiety did not reach a significant level in either boys or girls.

Mean scores of social anxiety for participants from each group (masculine, feminine, androgynous, or undifferentiated) were analyzed. The total score of the SADS as well as the social distress scale score were significantly higher in adolescents with undifferentiated gender role orientation. The lowest social anxiety scores were found in the group of masculine type.

\section{Perfectionism and hostility as personality factors of social anxiety}

A positive correlation was found between "concern over mistakes" $(\mathrm{p} \leq 0.05)$ and "overdoing" ( $\mathrm{p} \leq 0.001)$ on the Child Perfectionism Questionnaire (CPQ) subscales and SADS total score, as well as "social distress" and "social avoidance" subscales. The CPQ "high personal standards" subscale negatively correlated with the SADS "social avoidance" subscale $(\mathrm{p} \leq 0.05)$.

In order to test our hypothesis regarding the correlation between social anxiety and perfectionism, and based on the correlation found between perfectionism and social anxiety, a regression analysis was conducted. We regressed the dependent variable, social anxiety, on six Child Perfectionism Questionnaire subscales as independent variables.

As seen in Table 4, the SADS total score was positively associated with the parental perfectionism and overdoing subscales, and negatively associated with success as a main motive scale. Parental perfectionism explained a variance of .36 to social anxiety, overdoing .37 , and success -.48 , creating a total dispersion percent of $33 \%$.

Table 4. Results of multiple regression analyses with adolescents' levels of perfectionism parameters as independent variables and social anxiety as the dependent variable

\begin{tabular}{lcccc}
\hline \multicolumn{1}{c}{ Independent variables } & Dependent variable & $\mathbf{B}$ & $\mathbf{T}$ & $\mathbf{R}^{\mathbf{2}}$ \\
\hline High personal standards & & -.115 & -.792 & .431 \\
Parental perfectionism & & -.360 & 2.586 & .012 \\
Concern over mistakes & Social anxiety & -.009 & -.059 & .953 \\
Success as a main motive & & -.486 & -2.718 & .008 \\
Physical perfectionism & .145 & 1.057 & .294 \\
Overdoing & .370 & 3.154 & .002 \\
\hline
\end{tabular}

Correlational analysis found significant positive correlation between the hostility total score, perception of others as demonstrating superiority, perception of others as despising weakness, and parameters of social anxiety, as well as the SADS total score. 


\section{Family emotional communications and social anxiety}

Positive correlations were found between the social anxiety and suppression of emotions and outward well-being subscales, as well as the FEC total score. We can conclude that it is not common to discuss emotions; it is difficult to share negative experiences; and it is important to demonstrate for the families of socially anxious adolescents to put up a good front.

\section{Social anxiety and social support}

As expected, the total score of the Social Support Questionnaire and emotional support subscale was negatively correlated with the SADS total score and subscales. The practical support subscale was negatively correlated with the SADS total score.

\section{Discussion}

The results indicated that emotional disadaptation is present in our sample: $6 \%$ showed high scores for depressive symptoms (which is consistent with Volikova, Kholmogorova, \& Kalina, 2011), $12 \%$ of children mentioned suicidal thoughts in their self-report, and $6 \%$ declared that they are ready to commit suicide at any moment.

Moderate social anxiety was found in $63 \%$ of participants, medium anxiety in $30 \%$, and high social anxiety in $7 \%$. This study examined the relation between social anxiety and emotional disadaptation. Significant differences were found between depressive symptoms and the intensity of suicidal intentions and social anxiety: In groups with medium and high social anxiety, scores for depression were much higher than in groups with low SA.

Gender factors in social anxiety were studied as macrosocial ones. As was mentioned in the literature review, gender role orientation is one of the key elements of personal identity. It is identified according to the intensity of traditionally defined masculine and feminine characteristics. In our study, a prevalence of androgynous orientation in both boys and girls was found, which is consistent with other current empirical studies (Donnelly \& Twenge, 2016) and is undoubtedly typical for modern Western society.

No quantitative differences in social anxiety between girls and boys were found, which is consistent with similar studies (Garcia-Lopez, Ingles, \& Garcia-Fernandez, 2008). Our findings support the masculinity model, which alleges that the connection between psychological well-being and androgyny exists primarily due to the masculinity component of androgyny (Basoff \& Glass, 1982; Peleg, 2012; Moscovitch, Hofmann, \& Litz, 2005). According to this model, femininity does not contribute to psychological health, while masculine traits such as assertiveness, vigor, and aggression influence the reduction of social anxiety. On the other hand, people with a masculine role orientation may tend not to reflect and express their feelings connected with social fears and anxiety. The absence of a difference between social anxiety in boys and girls can also be explained by the fact that gender role orientations were distributed quite similarly in males and females. Social anxiety appears to be connected with gender role orientation, but not biological sex. 
Important and theoretically meaningful connections between social anxiety and different parameters of perfectionism were discovered. The "overdoing" and "concern over mistakes" scales positively correlated with both social avoidance and social distress. The "high personal standards" scale was negatively correlated with social anxiety. Regression analysis confirmed that high scores on such scales as "parental perfectionism" and "overdoing" and a low score on the "success as a main motive" scale have a significant effect on social anxiety. In summary, overdoing, concern over mistakes, and parental perfectionism contribute to high social anxiety, while high personal standards and success as main motives are connected to lower social anxiety. In our experience with the Moscow school system, odern children living in a big city tend to be concerned about their marks, spend most of their time doing homework, and therefore don't socialize with their peers; their parents are emotionally dependent on the children's marks. Changes in the educational system in Russia, the implementation of state exams, high levels of stress, and social expectations for academic achievement are typical. Overall we found support for the idea that there are two different dimensions of perfectionism contributing to social anxiety: concern over failure and high personal standards (success orientation).

Since perfectionism scales were first developed, researchers have argued that not all aspects of perfectionism are maladaptive, i.e., connected with high stress and social psychological disadaptation. Moreover, it was speculated that some dimensions of perfectionism may contribute to adaptation. According to D.M. Dunkley, D.C. Zuroff, and K.R. Blankstein (2006), the most destructive parameters are socially prescribed perfectionism and self-criticism. At the same time, high personal standards may be related to healthy functioning (Dibartolo, Frost, Chang, Lasota, \& Grills, 2004). Our results are in line with those found in the literature on social anxiety; the measure of personal standards had a weak negative relationship with social anxiety (Shumaker \& Rodebaugh, 2009); low personal standards and high evaluative concern were associated with the greatest social anxiety in adolescents (Levinson et. al., 2015).

In our research, such scales as "suppression of emotions" and "outward wellbeing" showed positive correlations with social anxiety. It can be speculated that in families of highly anxious children, it is not acceptable to discuss emotions, hard to share problems with other family members, emotional inhibition is rewarded, and there is an emphasis on demonstration of outward well-being.

In this study, social support was studied as an interpersonal factor of social anxiety. Analysis revealed significant negative correlations between the SADS total score (as well its subscales) and the social support scale total score and the emotional support subscale; the practical support scale was negatively correlated with the SADS total score. These findings confirm our hypothesis that socially anxious teens lack social support, especially emotional support.

\section{Conclusions}

In this paper we have presented a study of the psychological factors of social anxiety in adolescents. One third of the participants were found to be suffering from high social anxiety, which was connected with symptoms of depression, anxiety, 
and suicidal intentions. Social anxiety in adolescents was not related to biological sex but to gender role identity. Suppression of emotions and putting up a good front in the family had an impact on the SA of the children, as well as low social support and such personality traits as perfectionism and hostility.

These findings provide possible psychotherapy targets for treatment of social anxiety in teenagers. They indicate the need to work with parents' emotional schemas, beliefs related to the importance of showing outward well-being, and their perfectionistic standards for their children. Concern over mistakes and a behavioral strategy of overdoing are the targets for psychological assistance for children. Macrosocial factors need further investigation, in order to differentiate where these high standards come from.

\section{Limitations}

While it is acknowledged that other factors, including temperamental characteristics and genetic influences, contribute to social anxiety, they are not the focus of this paper.

\section{References}

Alden, L.E. (2005). Interpersonal perspectives on social phobia. In Ray Crozier \& Lynn E. Alden (Eds.) The Essential Handbook of Social Anxiety for Clinicians (pp. 167-192). UK: W. John Wiley \& Sons, Ltd.

Alden, L.E., \& Taylor, C.T. (2004). Interpersonal processes in social phobia. Clinical Psychology Review, 24, 857-882. doi:10.1016/j.cpr.2004.07.006

Bassoff E.S., \& Glass G.V. (1982). The relationship between sex roles and mental health: A meta-analysis of twenty-six studies. The Counseling Psychologist, 10, 105-112. doi: $10.1177 / 0011000082104019$

Bockstaele, B.V., \& Bögels, S.M. (2014). Social Anxiety (3rd ed.). New York: Academic Press. doi:10.1016/B978-0-12-394427-6.00025-X

Bruch, M.A., Fallon, M., \& Heimberg, R.G. (2003). Social phobia and difficulties in occupational adjustment. Journal of Counseling Psychology, 50, 109-117. doi:10.1037/0022-0167 .50 .1 .109

Clark, D.M., \& Wells, A.A. (1995). A cognitive model of social phobia. In R. Heimberg, M. Leibovits, D.A. Hope, \& F.R. Schneier (Eds.). Social Phobia: Diagnosis, Assessment and Treatment (pp. 69-93). New York: Guilford Press.

Dibartolo, P.M., Frost, R.O., Chang, P., Lasota, M., \& Grills, A.E. (2004). Shedding light on the relationship between personal standards and psychopathology: The case for contingent self-worth. Journal of Rational-Emotional \& Cognitive-Behavior Therapy, 22, 237-250. doi:10.1023/B:JORE.0000047310.94044.ac

Donnelly, K., \& Twenge, J.M. (2016). Masculine and feminine traits on the Bem Sex-Role Inventory, 1993-2012: A cross-temporal meta-analysis. Sex Roles, 1-10. doi:10.1007/s11199016-0625-y

Dryman, M. T., Gardner, S., Weeks, J. W., \& Heimberg, R. G., (2016). Social anxiety disorder and quality of life: How fears of negative and positive evaluation relate to specific domains of life satisfaction, Journal of Anxiety Disorders, 38, 1-8. doi: 10.1016/j.janxdis.2015.12.003

Dvoryanchikov, N.V., Nosov, S.S., \& Salamova, D.K. (2011). Polovoe samosoznanie i metody ego diagnostiki [Gender self-construal and its diagnostics]. Moscow: FLINTA; Moscow: Nauka. 
Dunkley, D.M., Zuroff, D.C., \& Blankstein, K.R. (2006). Specific perfectionism components versus self-criticism in predicting maladjustment. Personality and Individual Differences, 40(4), 665-676. doi:10.1016/j.paid.2005.08.008

Garanyan, N.G., Kholmogorova, A.B., \& Yudeeva, T.U. (2003). Vrazhdebnost kak lichnostnyi factor depressii i trevogi [Hostility as a personality factor for depression and anxiety]. (pp. 100114). Moscow: Institute of Psychology, Russian Academy of Sciences.

Garcia-Lopez, L.J., Ingles, C.J., \& Garcia-Fernandez, J.M. (2008). Exploring the relevance of gender and age differences in the assessment of social fears in adolescence. Social Behavior and Personality, 36, 385-390. doi: 10.2224/sbp.2008.36.3.385

Gilbert, P., \& Trower, P. (2001). Evolution and process in social anxiety. In W.R. Crozier \& L.E. Alden (Eds.) International handbook of social anxiety (pp. 259-279). Chichester: Wiley.

Hart, T.A., Turk, C.L., Heimberg, R.G., \& Liebowitz, M.R. (1999). Relation of marital status to social phobia severity. Depression and Anxiety, 10, 28-32. doi: 10.1002/(SICI)1520-6394(1999) 10:1<28::AID-DA5>3.0.CO;2-I

Heimberg, R.G., Brozovich, F.A., \& Rapee, R.M. (2014). Chapter 24 - A cognitive-behavioral model of social anxiety disorder, In Social Anxiety (3rd ed.), (pp. 705-28). San Diego: Academic Press. doi: 10.1016/B978-0-12-394427-6.00024-8

Hofmann, S.G. (2007). Cognitive factors that maintain social anxiety disorder: A comprehensive model and its treatment implications. Cognitive Behavior Therapy, 4, 193-209. doi: $10.1080 / 16506070701421313$

Hofmann, S.G., Asnaani, A., \& Hinton, D.E. (2010). Cultural aspects in social anxiety and social anxiety disorder. Depression and Anxiety, 27, 1117-1127. doi: 10.1002/da.20759

Kholmogorova, A.B. (2011). Integrativnaya psykhoterapiya rasstroistv affectivnogo spektra [Integrative psychotherapy of mood disorders]. Moscow: Medpraktika-M.

Kholmogorova, A.B., \& Petrova, G.A. (2007). Metody diagnostiki socialnoi podderzhki pri rasstroistvah affektivnogo spectra [Methods of social support diagnostics in patients with mood disorders]. In Meditsinskaya tekhnologiya [Medical technology]. Moscow: Roszdrav Research Institute of Psychiatry.

Kholmogorova, A.B., \& Volikova S.V. (2004). Semeinyi kontekst rasstroistv affectivnogo spectra [Family context of mood disorders]. Klinicheskaya i sotsialnaya psikhiatriya [Clinical and Social Psychiatry], 2, 43-56.

Krasnova, V.V. (2013). Sotsialnaya trevozhnost kak faktor narushenii interpersonalnykh otnoshenii i trudnostei $v$ uchebnoi deyatelnosti u studentov [Social anxiety as a factor of interpersonal problems and difficulties in learning activity in students]. Moscow: Moscow State University of Psychology and Education.

Kovacs, M. (1992). Children's Depression Inventory Manual. North Tonawanda, New York: MultiHealth Systems Inc.

Levinson, C.A., Rodebaugh, T.L., Shumaker, E.A., Menatti, A.R., Weeks, J.W., White, E.K., Heimberg, R.G., Warren, C.S., Blanco, C., Schneier, F., \& Liebowitz, M.R. (2015). Perception matters for clinical perfectionism and social anxiety. Journal of Anxiety Disorders, 29, 61-71. doi: 10.1016/j.janxdis.2014.11.002

Moscovitch, D.A., Hofmann, S.G., \& Litz, B.T. (2005). The impact of self-construals on social anxiety: A gender-specific interaction. Personality and Individual Differences, 8, 659-672. doi:10.1016/j.paid.2004.05.021.

Ollendick, T.H., \& Benoit, K.E. (2012). A parent-child interactional model of social anxiety disorder in youth. Clinical Child and Family Psychology Review, 15, 81-91. doi: 10.1007/ s10567-011-0108-1

Peleg, O. (2012). Social anxiety and social adaptation among adolescents at three age levels. Social Psychology of Education, 15, 207-218. doi: 10.1007/s11218-011-9164-0 
Schmidt, L.A., Polak, C.P., \& Spooner, A.L. (2005). Biological and environment contributions to childhood shyness: A diathesis-stress model. In W. Crozier and L. Alden (Eds.) The essential handbook of social anxiety for clinicians. New York: John Wiley \& Sons.

Schneier, F.R., Johnson, J., Hornig, C.D., Liebowitz, M.R., \& Weissman, M.M. (1992). Social phobia. Comorbidity and morbidity in an epidemiologic sample. Archives of General Psychiatry, 49, 282-288. doi:10.1001/archpsyc.1992.01820040034004

Shumaker, E.A., \& Rodebaugh, T.L. (2009). Perfectionism and social anxiety: Rethinking the role of high standards. Journal of Behavior Therapy and Experimental Psychiatry, 40(3), 423-433. doi:10.1016/j.jbtep.2009.04.002

Shikatani, B., Antony, M.M., Kuo, J.R., \& Cassin, S.E. (2014). The impact of social restructuring and mindfulness strategies on post event processing and affect in social anxiety disorder. Journal of Anxiety Disorders, 28, 570-579. doi: 10.1016/j.janxdis.

Vinas, F., Canals, J., Gras, M.E., Ros, C., \& Domenech-Llaberia, E. (2002). Psychological and family factors associated with suicidal ideation in pre-adolescents. Spanish Journal of Psychology, 5, 20-28. doi: 10.1017/S1138741600005795

Volikova, S.V., Kholmogorova, A.B., \& Kalina, O.G. (2011). Validizatsia oprosnika detskoi depressii M. Kovaka [Validation of M. Kovaks' child depression inventory]. Voprosy psikhologii [Issues in Psychology], 5, 121-132.

Volikova, S.V. (2012). Sovremennye issledovaniya detskogo perfektsionizma [Contemporary investigations of child perfectionism]. Sotsialnaya i klinicheskaya psikhiatriya [Social and Clinical Psychiatry], 22(2), 94-99.

Original manuscript received June 15, 2016

Revised manuscript accepted October 03, 2016

First published online June 30, 2017 